\title{
ON SOME FURTHER SECOND ORDER INTEGRAL INEQUALITIES
}

\author{
KATARZYNA WOJTECZEK-LASZCZAK
}

\begin{abstract}
Some weighted quadratic integral inequalities of the second order involving a function, its first and second derivative has been derived using the uniform method of obtaining integral inequalities. An example in which some new integral inequalities with Chebyshev weight functions appeared has been considered.
\end{abstract}

Mathematics subject classification (2000): 26D10.

Key words and phrases: Inequalities, higher order Hardy inequalities, absolutely continuous function, uniform method.

\section{REFERENCES}

[1] D.C. BENSON, Inequalities involving integrals of functions and their derivatives, J. Math. Anal. Appl.17 (1967), 292-308.

[2] S. EASWARAN, Quadratic functionals of $n$-th order, Canad. Math. Bull. 19 (1976), 159-167.

[3] B. Florkiewicz, M. Kuchta, Some quadratic integral inequalities of first order, Colloq. Math. 75 (1998), 7-18.

[4] B. Florkiewicz, A. Rybarski, Some integral inequalities of Sturm-Liouville type, Colloq. Math. 36 (1976), 127-141.

[5] B. Florkiewicz, K. WojteczeK, Some second order integral inequalities, in: Proceedings of the Third World Congress of Nonlinear Analysis Part 6 (Catania 2001). Nonlinear Analysis 47 (2001), 4307-4312.

[6] B. FloRKIEWICZ, K. WOJTECZEK, On somefurther Wirtinger-Beesackintegral inequalities, Demonstratio Mathematica 32 (1999), 495-502.

[7] B. FlorkiEWICZ, K. WoJTECZEK, Some second order integral inequalities of generalized Hardy type, Proc. Roy. Soc. Edinburgh Sec. A., 129 (1999), 947-958.

[8] G.H. Hardy, J.E. LitTLEWOOD, G. Polya, Inequalities, 2nd edn. Cambridge Univ. Press, Cambridge, 1991.

[9] B. OpIC, A. Kufner, Hardy-type Inequalities, Pitman Research Notes in Mathematics Series 219, Harlow, Essex, UK: Longman Scientific and Technical, 1990.

[10] A. KUFNER, - E. PERSSON, Weighted inequalities of Hardy type, World Scientific, New Jersey-LondonSingapore-Hongkong, 2003.

[11] A. Kufner, G. Sinnamon, Overdetermined Hardy inequalities, J. Math. Anal. Appl. 213 (1997), 468-486.

[12] A. KufNeR, A. WANNEBO, Some remarks on the Hardy inequality for higher order derivatives, in: General Inequalities, 6, International Series of Numerical Mathematics 103, ed. W. Walter, Basel: Birkhäuser, (1992), 33-48.

[13] M.K. Kwong, A. ZeTtL, Norm Inequalities for Derivatives and Differences, Springer - Verlag, Berlin, 1992.

[14] W. Leighton, Quadratic Functional of second order, Trans. Amer. Math. Soc., 151 (1970), 309-322.

[15] D.S. Mitrinović, J.E. PeČARIĆ, A.M. FInK, Inequalities Involving Functions and Their Integrals and Derivatives, Kluwer Acad. Publ., Dordrecht, 1991.

[16] M. NASYROVA, V.D. STEPANOV, On weighted Hardy inequalities on semiaxis for functions vanishing at the endpoints J. Inequal. Appl. 3 (1970), 223-238.

[17] G. TALenti, Una disuguaglianza fra $u, u^{\prime}, u^{\prime \prime}$, Boll. Un. Mat. Ital. 4, 11, (1975), 375-388. 